\title{
On PID Controller Design by Combining Pole Placement Technique with Symmetrical Optimum Criterion
}

\author{
Viorel Nicolau \\ "Dunarea de Jos" University of Galati, 47 Domneasca Street, 800008 Galati, Romania \\ Correspondence should be addressed to Viorel Nicolau; viorel.nicolau@ugal.ro
}

Received 10 April 2013; Revised 8 July 2013; Accepted 8 July 2013

Academic Editor: Cristian Toma

Copyright (C) 2013 Viorel Nicolau. This is an open access article distributed under the Creative Commons Attribution License, which permits unrestricted use, distribution, and reproduction in any medium, provided the original work is properly cited.

\begin{abstract}
In this paper, aspects of analytical design of PID controllers are studied, by combining pole placement technique with symmetrical optimum criterion. The proposed method is based on low-order plant model with pure integrator, and it can be used for both fast and slow processes. Starting from the desired closed-loop transfer function, which contains a second-order oscillating system and a lead-lag compensator, it is shown that the zero value depends on the real-pole value of closed-loop transfer function. In addition, there is only one pole value, which satisfies the assumptions of symmetrical optimum criterion imposed to open-loop transfer function. In these conditions, by combining the pole placement technique with symmetrical optimum criterion, the analytical expressions of the controller parameters can be simplified. For simulations, PID autopilot design for heading control problem of a conventional ship is considered.
\end{abstract}

\section{Introduction}

PID controllers are widespread in industry, being the most popular ones for decades. Hence, even small improvements in PID design could have major impact worldwide [1]. There are many theoretical and practical papers on PID tuning algorithms, in time and frequency domain. They are using analytical [1-5], graphical [6-8], or empirical methods, including artificial intelligence $[9,10]$.

Analytical methods rely on low-order plant models characterized by small number of parameters. Two reduced plant models are mostly used: pure integrator plus firstorder model, like those for thermal and electromechanical processes, and the first-order plus dead-time model, like in chemical processes, respectively [11].

The proposed analytical method in the paper is based on low-order plant model with pure integrator, and it can be used for both fast and slow processes. An application example is discussed for autopilot design of conventional ships in course-keeping or course-changing control problems.
Pole placement technique (PPT) [12-14] and symmetrical optimum criterion (SOC) [15-18] were intensively studied as separated methods on both types of reduced plant models mentioned above.

If PPT is used to synthesize the PID controller, the first step is to specify some performance conditions of the closedloop system, which lead to the analytical expression of the closed-loop transfer function (CLTF). The PID parameters depend on the CLTF and plant model parameters. In general, the closed-loop system is approximated by a second order reference model. If the plant model has pure integrator element and PID type is desired for the controller, then the CLTF must contain an additional lead-lag compensator. In this case, the reference model has four parameters, two from the second order reference model and the other two from the compensator. All these parameters are present in the analytical expressions of PID controller. In addition, if the well known symmetrical optimum criterion [6] is used, the parameters of compensator have particular values, as stated in the paper, which simplify the PID parameters. 
By combining the pole placement technique with symmetrical optimum criterion, the analytical expressions of the PID parameters depend only on the parameters of the plant and second order reference models.

In this paper, PID autopilot design for heading control problem of a conventional ship is considered in simulations. The ship model is obtained from the equations describing the horizontal motion of the ship, expressing conservation of hydrodynamic forces and moments. For ship dynamics modeling, which characterize a slow process, the first type of reduced model is used, with pure integrator plus first-order model.

The autopilot must assure performance conditions in both course-keeping and course-changing control problems. It must be PID type to assure null stationary error for both step and ramp variations on reference and disturbances inputs $[19,20]$. Thus, in course-keeping control, the PID autopilot can compensate the stationary error (e.g., drift effect), generated by constant or slowly time-variable disturbances (e.g., wind, sea currents). Also, in course-changing control problems, PID autopilot can act as a tracking system to a desired trajectory, consisting of line segments [21].

As a result, the reference model contains a second-order oscillating system and a lead-lag compensator, with real negative values for pole-zero pair, and the open-loop transfer function (OLTF) contains a double-integrator element.

The goal of this paper is to find the simplified analytical expressions of PID controller parameters, which satisfy two simultaneous conditions: the desired behavior of closed-loop system and symmetrical characteristics of the open-loop transfer function. Applying PPT, the controller parameters depend on the parameters of the plant and closed-loop transfer functions. The resulting open-loop transfer function contains a double-integrator element and a pole-zero pair, with real negative values. Hence, the symmetrical optimum criterion can be used. Imposing symmetrical characteristics of the open-loop transfer function, which can be obtained in certain conditions stated in the paper, the analytical expressions of the controller parameters can be simplified.

The paper is organized as follows. Section 2 provides mathematical models for the plant, PID controller, and reference closed-loop system used in simulations, for both fast and slow processes. In Section 3, the PID controller synthesis using PPT is presented. The analytical expressions of controller parameters are simplified in Section 4, by imposing symmetrical characteristics of OLTF. Section 5 describes the simulation results, using a PID autopilot for heading control of a ship. Conclusions are presented in Section 6.

\section{Mathematical Models}

Mathematical models for plant, PID controller, and reference closed-loop system used in simulations are discussed in this section. The classical factorized forms of transfer functions are considered, to express poles and zeros, based on which pole placement technique and symmetrical optimum criterion can be used.
In the paper, a low-order plant model with pure integrator is considered, for both fast and slow processes. The controller is of PID type, being imposed by performance conditions of closed-loop system, which are detailed at the end of this section.

The classical structure of the control loop, for a linear SISO process without disturbances, is illustrated in Figure 1.

The plant has a low-order model, which contains a pure integrator. It is characterized by a dominant time constant $\left(T_{P}\right)$ and a gain coefficient $\left(k_{P}\right)$. The analytical expressions of plant model and PID controller depend on the process type, fast or slow [22].

(a) If the process is fast, then the small time constants can not be neglected. An equivalent small time constant $\left(T_{\Sigma}\right)$ is added to the model, corresponding to the sum of parasitic time constants:

$$
H_{P}(s)=\frac{k_{P}}{s \cdot\left(s T_{P}+1\right) \cdot\left(s T_{\Sigma}+1\right)},
$$

where $T_{\Sigma}<T_{P}$.

In this case, the PID controller is of the following form:

$$
H_{C}(s)=\frac{k_{C}}{s T_{C}} \cdot\left(s T_{C}+1\right) \cdot\left(s T_{C}^{\prime}+1\right),
$$

where $T_{\Sigma}<T_{C}^{\prime}<T_{C}$.

The open-loop transfer function, $H(s)=H_{C}(s) \cdot H_{P}(s)$, is as follows:

$$
\begin{aligned}
H(s)= & \frac{k_{C}}{s T_{C}} \cdot\left(s T_{C}+1\right) \cdot\left(s T_{C}^{\prime}+1\right) \\
& \cdot \frac{k_{P}}{s \cdot\left(s T_{P}+1\right) \cdot\left(s T_{\Sigma}+1\right)} .
\end{aligned}
$$

Using pole cancellation, the nonzero dominant pole of the plant model is cancelled by choosing $T_{C}^{\prime}=T_{P}$. Thus, only two controller parameters must be determined: $k_{C}$ and $T_{C}$.

It can be observed that if the process does not have any non-zero dominant pole (model without time constant $T_{P}$ ), then the controller is of PI type (without time constant $T_{C}^{\prime}$ ), and the same parameters must be determined $\left(k_{C}\right.$ and $\left.T_{C}\right)$.

(b) If the process is slow, the equivalent small time constant can be neglected $\left(T_{\Sigma} \ll T_{P}\right)$ and the plant model is as follows:

$$
H_{P}(s)=\frac{k_{P}}{s \cdot\left(s T_{P}+1\right)} .
$$

In this case, the PID controller contains a supplementary degree of freedom and it is of the following form:

$$
H_{C}(s)=\frac{k_{C}}{s T_{C}} \cdot\left(s T_{C}+1\right) \cdot \frac{s T_{C}^{\prime}+1}{s T_{1}+1},
$$

where $T_{1}<T_{C}^{\prime}<T_{C}$. follows:

The open-loop transfer function for slow process is as

$$
H(s)=\frac{k_{C}}{s T_{C}} \cdot\left(s T_{C}+1\right) \cdot \frac{s T_{C}^{\prime}+1}{s T_{1}+1} \cdot \frac{k_{P}}{s \cdot\left(s T_{P}+1\right)} .
$$




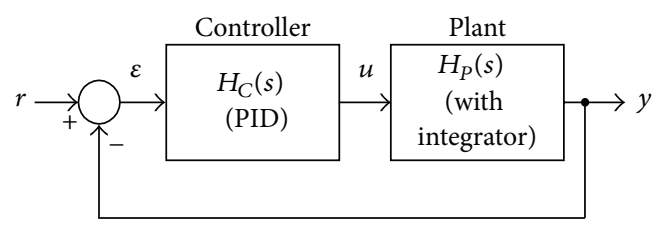

Figure 1: Classical structure of the control loop.

Again, the non-zero dominant pole of the plant model is cancelled, using pole cancellation, resulting $T_{C}^{\prime}=T_{P}$. In this case, three controller parameters must be determined: $k_{C}, T_{C}$, and $T_{1}$. This is a more general case, due to the time constant $T_{1}$, which is not imposed by the process and it can be chosen. Therefore, in this paper, the plant model given in (4) is used for simulations, but discussions include both plant models given in (1) and (4).

The open-loop transfer functions, given in (3) and (6), have similar expressions, after pole cancellation:

$$
H(s)=H_{C}(s) \cdot H_{P}(s)=\frac{k_{C} \cdot k_{P} \cdot\left(s T_{C}+1\right)}{s^{2} T_{C} \cdot(s T+1)},
$$

where the time constant $T$ has different meanings, depending on the type of process considered. In the first case for fast processes, it represents the equivalent small time constant imposed by the process $\left(T=T_{\Sigma}\right)$, while in the second case it is a controller parameter $\left(T=T_{1}\right)$.

In general, the behavior of the closed-loop system is approximated by a second order reference model:

$$
H_{0}(s)=\frac{\omega_{0}^{2}}{s^{2}+2 \zeta \omega_{0} s+\omega_{0}^{2}},
$$

where $\omega_{0}>0$ is the natural frequency and $\zeta>0$ is the damping coefficient. The performance conditions of the closed-loop system can be specified, by imposing the model parameters $\left(\zeta\right.$ and $\left.\omega_{0}\right)$.

Starting from the plant model with pure integrator and considering the reference model from (8), it results a real-PD controller. The corresponding OLTF is as follows:

$$
H(s)=H_{C}(s) \cdot H_{P}(s)=\frac{H_{0}(s)}{1-H_{0}(s)}=\frac{\omega_{0}^{2}}{s\left(s+2 \zeta \omega_{0}\right)} .
$$

It can be observed that the open-loop transfer function, corresponding to CLTF presented in (8), has one pure integrator, which assures null stationary error only for step variations of reference input, denoted $r$ in Figure 1.

If the controller must assure null stationary error for both step and ramp variations on reference and disturbances inputs, then the controller must be PID type. In this case, the open-loop transfer function contains a double-integrator element, one being included from the plant model, and the other one from the PID controller. The resulted OLTF has the general form expressed in (7), which cannot be generated from the reference model given in (8).

To obtain the expression of OLTF given in (7), the reference model must be completed with a lead-lag compensator, which contains a pole-zero pair, with real and negative values [22]:

$$
H_{0}(s)=\frac{k_{0} \cdot(s+z)}{\left(s^{2}+2 \zeta \omega_{0} s+\omega_{0}^{2}\right) \cdot(s+p)},
$$

where $z>0, p>0$, and $k_{0}=\omega_{0}^{2} \cdot p / z$, so that $\left|H_{0}(0)\right|=1$.

Particular values for pole-zero pair of lead-lag compensator simplify the analytical expressions of the controller parameters, as stated in the next sections. In addition, symmetrical characteristics of OLTF are obtained.

\section{Aspects of PID Controller Design Using Pole Placement Technique}

In this section, existence conditions of parameters of CLTF are discussed, and PID parameters are determined based on PPT [19]. Consider the control structure shown in Figure 1, with the OLTF given in (7) and the desired CLTF in (10).

Proposition 1. For every value of the pole $(s=-p)$ in the desired closed-loop transfer function given in (10), there is only one zero value $(s=-z)$ for which the open-loop transfer function has a double-pole in origin, and in addition, the zero frequency is smaller than the pole frequency: $z<p$.

Proof. The proposition demonstration includes the next lemma results.

Lemma 2. The necessary and sufficient condition, for the existence of a double-pole in origin for the open-loop transfer function of a control system illustrated in Figure 1, starting from a desired CLTF of the form given in (10), is as follows:

$$
2 \zeta p z+\omega_{0} z-\omega_{0} p=0 .
$$

Proof. The transfer function of the open-loop system can be computed starting from the desired CLTF:

$$
\begin{aligned}
H(s) & =H_{C}(s) \cdot H_{P}(s)=\frac{H_{0}(s)}{1-H_{0}(s)} \\
& =\frac{\omega_{0}^{2} \cdot p / z \cdot(s+z)}{s^{3}+s^{2}\left(p+2 \zeta \omega_{0}\right)+s\left[2 \zeta \omega_{0} p+\omega_{0}^{2}(1-p / z)\right]} .
\end{aligned}
$$

From (12) it can be observed that a double-pole in origin is obtained for every frequency, if and only if the coefficient of the third term of denominator is null:

$$
2 \zeta \omega_{0} p+\omega_{0}^{2}\left(1-\frac{p}{z}\right)=0
$$

which is equivalent with equation in (11) for $\omega_{0} \neq 0$.

As a result, the unique zero value results, whose expression depends on the selected pole value:

$$
s=-z=-\frac{\omega_{0} p}{2 \zeta p+\omega_{0}}, \quad \forall p, \omega_{0}, \zeta>0
$$


For every frequency $(p)$ of the pole, the corresponding frequency $(z)$ of the zero given in (14) is smaller:

$$
z=\frac{\omega_{0} p}{2 \zeta p+\omega_{0}}<p, \quad \forall p, \omega_{0}, \zeta>0 .
$$

In this case, the real values of pole-zero pair and conjugate complex poles, of the desired CLTF given in (10), are illustrated in the complex plane in Figure 2.

Using (13) and (15) in (12), the expression of the open-loop transfer function is obtained:

$$
H(s)=\frac{\omega_{0} \cdot\left(2 \zeta p+\omega_{0}\right) \cdot\left(s+\left(\omega_{0} p /\left(2 \zeta p+\omega_{0}\right)\right)\right)}{s^{2} \cdot\left[s+\left(p+2 \zeta \omega_{0}\right)\right]} .
$$

The zero and pole frequencies of the open-loop transfer function are denoted by $\omega_{z}$ and $\omega_{p}$, respectively:

$$
\omega_{z}=\frac{\omega_{0} p}{2 \zeta p+\omega_{0}}=z, \quad \omega_{p}=2 \zeta \omega_{0}+p
$$
follows:

The open-loop transfer function can be rewritten as

$$
H(s)=\frac{\omega_{0}\left(2 \zeta p+\omega_{0}\right) \cdot\left(s+\omega_{z}\right)}{s^{2} \cdot\left[s+\omega_{p}\right]} .
$$

Putting into evidence the time constants, the open-loop transfer function can be rewritten, like in (7):

$$
H(s)=\frac{\omega_{0}^{2} p \cdot\left(s\left(\left(2 \zeta p+\omega_{0}\right) / \omega_{0} p\right)+1\right)}{s^{2}\left(2 \zeta \omega_{0}+p\right)\left(s\left(1 /\left(2 \zeta \omega_{0}+p\right)\right)+1\right)} .
$$

Equaling OLTF without pole cancellation, from (3) or (7), with (19), an equality of two polynomials of 3rd order in $s$ variable is obtained:

$$
\begin{aligned}
& k_{P} \cdot k_{C} \cdot\left(2 \zeta \omega_{0}+p\right) \cdot\left(s T_{C}+1\right) \cdot\left(s T_{C}^{\prime}+1\right) \\
& \cdot\left(s \frac{1}{2 \zeta \omega_{0}+p}+1\right) \\
& =T_{C} \cdot \omega_{0}^{2} p \cdot(s T+1) \cdot\left(s T_{P}+1\right) \cdot\left(s \frac{2 \zeta p+\omega_{0}}{\omega_{0} p}+1\right),
\end{aligned}
$$

where $T=T_{\Sigma}$ or $T=T_{1}$, depending on the plant model.

Because the equality must be true for every frequency, it results a four equation system:

$$
\begin{gathered}
k_{P} \cdot k_{C} \cdot\left(2 \zeta \omega_{0}+p\right)=T_{C} \cdot \omega_{0}^{2} p, \\
T_{C} \cdot T_{C}^{\prime} \cdot \frac{1}{2 \zeta \omega_{0}+p}=T \cdot T_{P} \cdot \frac{2 \zeta p+\omega_{0}}{\omega_{0} p}, \\
T_{C} \cdot T_{C}^{\prime}+\left(T_{C}+T_{C}^{\prime}\right) \cdot \frac{1}{2 \zeta \omega_{0}+p} \\
=T \cdot T_{P}+\left(T+T_{P}\right) \cdot \frac{2 \zeta p+\omega_{0}}{\omega_{0} p}, \\
T_{C}+T_{C}^{\prime}+\frac{1}{2 \zeta \omega_{0}+p}=T+T_{P}+\frac{2 \zeta p+\omega_{0}}{\omega_{0} p} .
\end{gathered}
$$

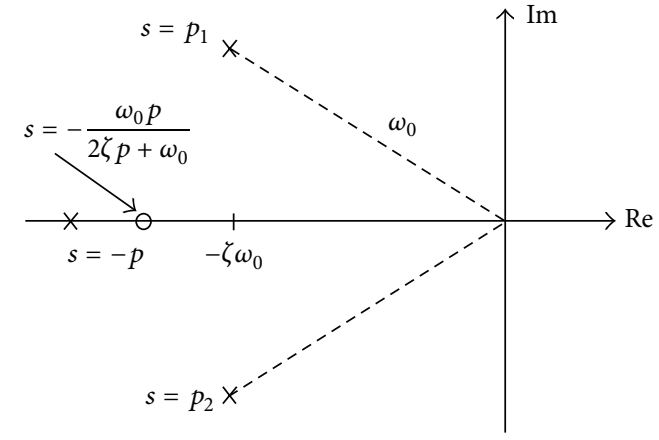

Figure 2: Poles and zero of desired CLTF given in (10).

The solutions of the equation system are the PID controller parameters [19]:

$$
\begin{gathered}
k_{C}=\frac{\omega_{0}}{k_{P}} \cdot \frac{2 \zeta p+\omega_{0}}{2 \zeta \omega_{0}+p}, \\
T_{C}=\frac{2 \zeta p+\omega_{0}}{\omega_{0} p}=\frac{1}{\omega_{z}}, \\
T_{C}^{\prime}=T_{P}, \\
T=\frac{1}{2 \zeta \omega_{0}+p}=\frac{1}{\omega_{p}} .
\end{gathered}
$$

It can be observed that the solution (22c) represents the pole cancellation condition, and it does not depend on the $p$ pole value.

If a fast process is considered, then the time constant $T$ given in (22d) represents the equivalent small time constant of the plant model $\left(T=T_{\Sigma}\right)$. If the process is slow, then the same time constant $T$ is a controller parameter $\left(T=T_{1}\right)$.

The time constants must satisfy the inequalities:

$$
T<T_{C}^{\prime}<T_{C}
$$

This can be transposed into frequency domain:

$$
\omega_{z}<\frac{1}{T_{P}}<\omega_{p}
$$

Using (17) in (24), a system of 2 inequalities is obtained:

$$
\begin{gathered}
2 \zeta \omega_{0}+p>\frac{1}{T_{P}}, \\
2 \zeta p+\omega_{0}>p \omega_{0} T_{P} .
\end{gathered}
$$

The reference model must be chosen so that the parameters of closed-loop transfer function $\left(\omega_{0}, \zeta\right.$, and $\left.p\right)$ satisfy this system of inequalities.

\section{Design Aspects by Imposing Symmetrical Characteristics of OLTF}

After applying PPT, the parameters of PID controller depend on the CLTF parameters $\left(\omega_{0}, \zeta\right.$, and $\left.p\right)$. In general, $\omega_{0}$ and $\zeta$ 
characterize the desired behavior of the closed-loop system, and they have fixed values, while the pole value can be chosen. Specific pole values can be imposed by using supplementary conditions. In this paper, the conditions for choosing the pole value refer to the symmetrical optimum criterion, which simplify the expressions of PID parameters.

The goal is to find that pole value of the CLTF, which satisfies the assumptions of symmetrical optimum criterion around natural frequency $\omega_{0}$, for the transfer function of open-loop system given in (16). Using this value, the expressions of PID parameters given in (22a), (22b), (22c), and (22d) are simplified.

Proposition 3. There is only one admissible value for the pole $(s=-p)$ of CLTF given in (10), so that the corresponding OLTF given in (16) has symmetrical characteristics around $\omega_{0}$, and this value is as follows:

$$
p=\omega_{0}, \quad \forall p, \omega_{0}, \zeta>0 .
$$

Proof. For the specified open-loop transfer function, only the symmetry of magnitude-frequency characteristic around natural frequency $\omega_{0}$ must be imposed, as it implies also the symmetry of phase-frequency characteristic.

The general form of the symmetrical optimum criterion imposes two conditions for magnitude-frequency characteristic of OLTF:

(a) cross-over frequency $\omega_{0}$ must be equally placed between zero and pole frequencies on the 10-base logarithmic scale:

$$
\frac{\omega_{0}}{\omega_{z}}=\frac{\omega_{p}}{\omega_{0}}
$$

(b) for cross-over frequency $\omega_{0}$, the magnitude-frequency characteristic of OLTF must have $0 \mathrm{~dB}$ :

$$
\left|H\left(j \omega_{0}\right)\right|=1 .
$$

Using (17) in (27), the first condition in $p$ variable is as follows:

$$
\frac{\omega_{0} p}{2 \zeta p+\omega_{0}} \cdot\left(2 \zeta \omega_{0}+p\right)=\omega_{0}^{2} \Longleftrightarrow p^{2}=\omega_{0}^{2}
$$

From (29), the solution results are as follows:

$$
p=\omega_{0}, \quad \forall p, \omega_{0}, \zeta>0 .
$$

Therefore, the condition given in (27) is satisfied if and only if $p=\omega_{0}$.

For the second condition in (28), the magnitude of openloop transfer function in frequency $\omega_{0}$ is computed from (18):

$$
\left|H\left(j \omega_{0}\right)\right|=\frac{\left(2 \zeta p+\omega_{0}\right) \cdot \sqrt{\omega_{z}^{2}+\omega_{0}^{2}}}{\omega_{0} \cdot \sqrt{\omega_{p}^{2}+\omega_{0}^{2}}} .
$$

The frequencies $\omega_{z}$ and $\omega_{P}$ are replaced with their expressions from (17), resulting the following:

$$
\left|H\left(j \omega_{0}\right)\right|=\sqrt{\frac{p^{2}+\omega_{0}^{2}+4 \zeta p \omega_{0}+4 \zeta^{2} p^{2}}{p^{2}+\omega_{0}^{2}+4 \zeta p \omega_{0}+4 \zeta^{2} \omega_{0}^{2}}} .
$$

Using (32) in (28), the same solution in (30) is obtained: $p^{2}=\omega_{0}^{2} \Leftrightarrow p=\omega_{0}, \forall p, \omega_{0}, \zeta>0$.

Using (30) in (15), it results the following:

$$
p=\omega_{0} \Longrightarrow z=\frac{\omega_{0}}{2 \zeta+1} \text {. }
$$

The expression of CLTF becomes

$$
H_{0}(s)=\frac{\omega_{0}^{2} \cdot(2 \zeta+1) \cdot\left(s+\left(\omega_{0} /(2 \zeta+1)\right)\right)}{\left(s^{2}+2 \zeta \omega_{0} s+\omega_{0}^{2}\right) \cdot\left(s+\omega_{0}\right)} .
$$

From (17), the zero and pole frequencies of OLTF are

$$
\omega_{z}=\frac{\omega_{0}}{2 \zeta+1}, \quad \omega_{p}=\omega_{0} \cdot(2 \zeta+1) .
$$
follows:

The open-loop transfer function can be rewritten as

$$
H(s)=\frac{\omega_{0}^{2}(2 \zeta+1) \cdot\left(s+\left(\omega_{0} / 2 \zeta+1\right)\right)}{s^{2} \cdot\left[s+\omega_{0}(2 \zeta+1)\right]} .
$$

The real values of pole-zero pair and complex conjugate poles, of the CLTF given in (34), are illustrated in Figure 3.

The position of the zero, $s=-z$, depends on $\zeta$ parameter. Three situations are possible:

(i) if $\zeta \in(0,1 / 2)$, then $-\omega_{0}<-z<-\zeta \omega_{0}$ and the zero is placed between the two points: $s=-\omega_{0}$ and $s=-\zeta \omega_{0}$, respectively;

(ii) if $\zeta=1 / 2$, then $-z=-\zeta \omega_{0}$. This is the particular case of the Kessler's symmetrical optimum method [6];

(iii) if $\zeta>1 / 2$, then $0>-z>-\zeta \omega_{0}$ and the zero is placed to the right of the point $s=-\zeta \omega_{0}$. This is the case illustrated in Figure 3.

Knowing the pole value of CLTF, $p=\omega_{0}$, the simplified parameters of PID controller result from (22a), (22b), (22c), and (22d):

$$
\begin{gathered}
k_{C}=\frac{\omega_{0}}{k_{P}}, \\
T_{C}=\frac{2 \zeta+1}{\omega_{0}}, \\
T_{C}^{\prime}=T_{P}, \\
T=\frac{1}{(2 \zeta+1) \cdot \omega_{0}} .
\end{gathered}
$$

In this case, the system of inequalities from (25a) and (25b) is simpler, including only 2 variables from reference model $\left(\omega_{0}\right.$ and $\left.\zeta\right)$ :

$$
\begin{gathered}
(2 \zeta+1) \cdot \omega_{0}>\frac{1}{T_{P}}, \\
\frac{2 \zeta+1}{\omega_{0}}>T_{P} .
\end{gathered}
$$




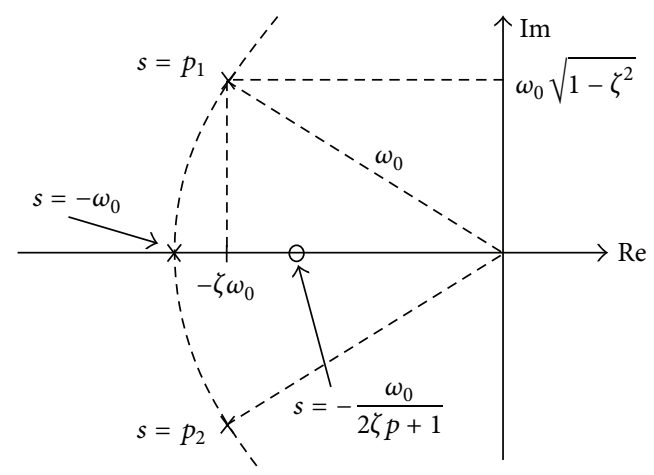

Figure 3: Poles and zero of CLTF for $p=\omega_{0}$.

Proposition 4. In the case of symmetrical characteristics of the OLTF given in (36) around the natural frequency $\omega_{0}$, the phase margin and the distance between the frequency points on the 10-base logarithmic scale depend only on the parameter $\zeta$.

Proof. The distance between the frequency points on the logarithmic scale can be easily obtained, using (35) in (27):

$$
\frac{\omega_{0}}{\omega_{z}}=\frac{\omega_{p}}{\omega_{0}}=2 \zeta+1
$$
follows:

Using the OLTF given in (36), the phase margin results as

$$
\varphi_{m}=\pi+\arg \left(H\left(j \omega_{0}\right)\right)=\operatorname{arctg}(2 \zeta+1)-\operatorname{arctg}\left(\frac{1}{2 \zeta+1}\right) .
$$

For particular case of the Kessler's symmetrical optimum method $(\zeta=0.5)$, the distance between frequency points is equal with an octave and the phase margin is $\varphi_{m}=$ 36.87 [deg].

\section{Simulation Results}

For simulations, the heading control problem of a ship is considered, using a PID autopilot.

The ship yaw angle $(\psi)$ is the model output, and rudder command $(\delta)$ generated by autopilot is the control input. The ship model is linear with parametric uncertainties, depending on ship loading conditions and forward speed of the ship, while the wave characteristics change frequently.

It is a first order Nomoto model of the form given in (4), being identified for a ship speed of 22 knots [21]:

$$
H_{P}(s)=\frac{\psi(s)}{\delta(s)}=\frac{k_{P}}{s \cdot\left(s T_{P}+1\right)}
$$

where $\psi(s)$ and $\delta(s)$ represent the Laplace transforms of yaw angle and rudder angle, respectively.

The ship model parameters are as follows:

$$
k_{P}=-0.0834\left[\mathrm{~s}^{-1}\right], \quad T_{P}=5.98[\mathrm{~s}] .
$$

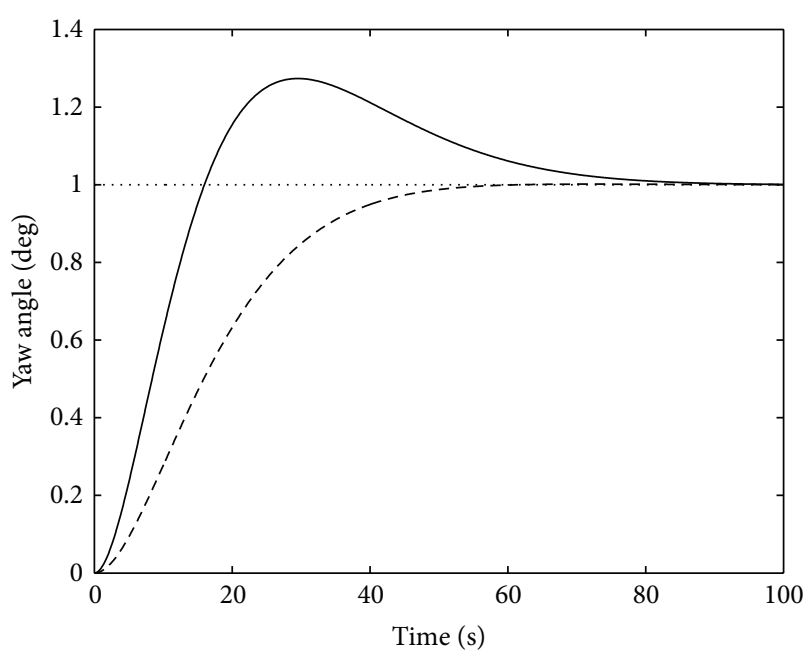

FIGURE 4: Step response of the closed-loop system.

The autopilot model is given in (5) and the desired CLTF is given in (10). The parameters $\omega_{0}$ and $\zeta$ are chosen from performance conditions [20]:

$$
\zeta=0.9, \quad \omega_{0}=0.1[\mathrm{rad} / \mathrm{s}] .
$$

Using the values from (42) and (43), it can be easily verified that the conditions in (38a) and (38b) are satisfied.

From the desired closed-loop transfer function with pole-zero pair specified in (30) and (33), and imposing symmetrical characteristics of the OLTF, the expressions in (34) and (36) are obtained. From (37a), (37b), (37c), and (37d), the autopilot parameters are obtained:

$$
\begin{array}{cc}
k_{C}=-1.2, & T_{C}=28[\mathrm{~s}], \\
T_{C}^{\prime}=5.98[\mathrm{~s}], & T_{1}=3.57[\mathrm{~s}] .
\end{array}
$$

The step response of the closed-loop transfer function is illustrated in Figure 4. The step reference input is represented with dotted line.

Considering the second order reference model given in (8), the resulting autopilot is of real-PD type. The response is over-dumped, being represented with dashed line. For PID autopilot based on reference model with lead-lag compensator given in (10), the response is overshot and it is drawn with continuous line.

Similarly, the ramp response of the CLTF is illustrated in Figure 5. The ramp reference input is represented with dotted line. Considering the second order reference model given in (8) with real-PD type, the response is shown with dashed line. An important stationary error can be observed. For reference model with lead-lag compensator given in (10) with PID autopilot, the response is represented with continuous line. In this case, a null stationary error is obtained.

PID autopilot assures null stationary error for both step and ramp variations on reference inputs.

For reference model with compensator and PID autopilot, the symmetrical characteristics of the open-loop transfer 


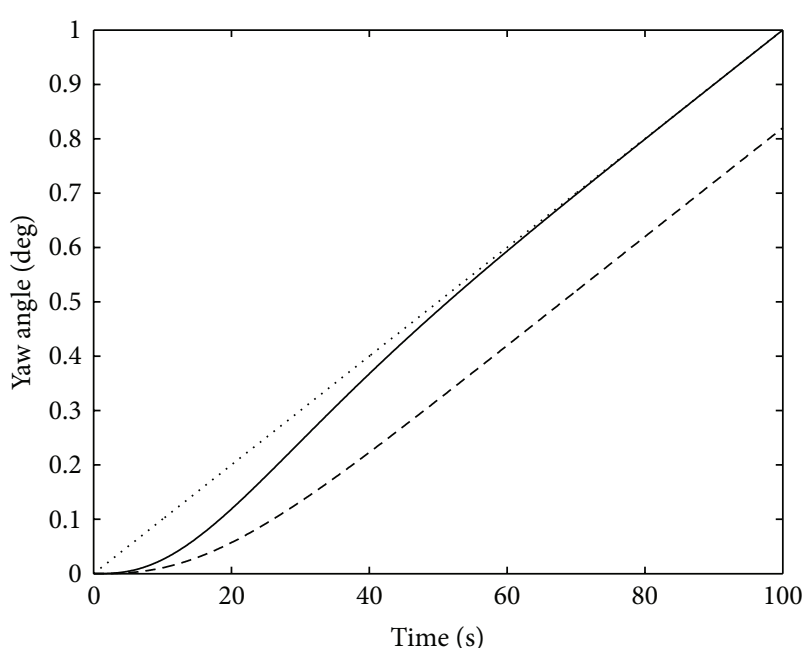

FIGURE 5: Ramp response of the closed-loop system.

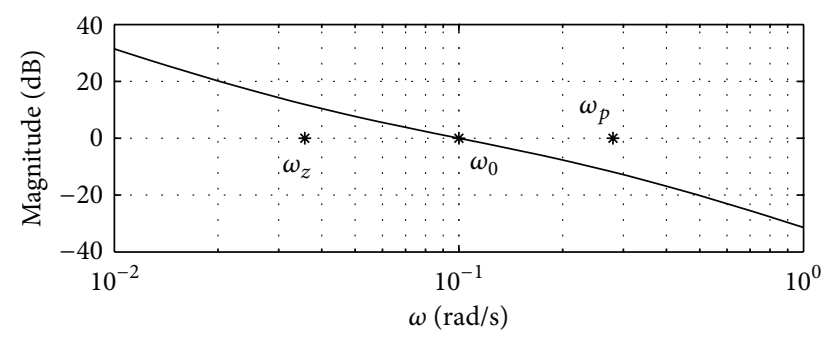

(a)

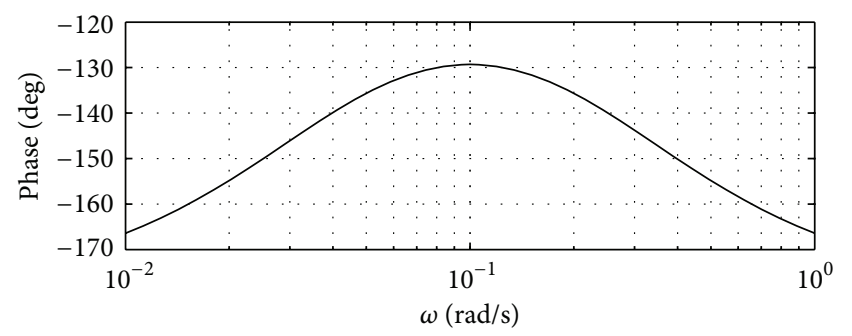

(b)

FIgURE 6: Symmetrical characteristics of OLTF.

function are illustrated in Figure 6. It can be observed that the two conditions for magnitude-frequency characteristic of OLTF generate the symmetry of phase-frequency characteristic. In this case, the phase margin is $\varphi_{m}=50.69$ [deg].

\section{Conclusions}

By combining the pole placement technique with symmetrical optimum criterion, the analytical expressions of PID parameters are simplified. In addition, it was demonstrated that there is only one possible pair for the pole-zero values of closed-loop transfer function, so that the PID controller satisfied two simultaneous conditions: the desired behavior of closed-loop system and symmetrical characteristics of the open-loop transfer function. For simulations, PID autopilot design for heading control problems of a conventional ship is considered. PID autopilot assures null stationary error for both step and ramp variations on reference inputs.

\section{References}

[1] G. J. Suva, A. Datta, and S. P. Bhattacharyya, "New results on the synthesis of PID controllers," IEEE Transactions on Automatic Control, vol. 47, no. 2, pp. 241-252, 2002.

[2] J. I. Yuz and M. E. Salgado, "From classical to state-feedbackbased controllers," IEEE Control Systems Magazine, vol. 23, no. 4, pp. 58-67, 2003.

[3] S. Sujitjorn and W. Wiboonjaroen, "State-PID feedback for pole placement of LTI systems," Mathematical Problems in Engineering, vol. 2011, Article ID 929430, 20 pages, 2011.

[4] G. Herjólfsson and A. Soffía Hauksdóttir, "Direct computation of optimal PID controllers," in Proceedings of the 42nd IEEE Conference on Decision and Control, pp. 1120-1125, December 2003.

[5] K. J. Astrom and T. Hagglund, PID Controllers: Theory, Design, and Tuning, Instrument Society of America, Research Triangle Park, NC, USA, 1995.

[6] C. Kessler, "Das symmetrische optimum," Regelungstechnik, vol. 6, pp. 395-400 and 432-436, 1958.

[7] K. J. Astrom and T. Hagglund, "Automatic tuning of simple regulators with specifications on phase and amplitude margins," Automattca, vol. 20, no. 5, pp. 645-651, 1984.

[8] Y. Tang and R. Ortega, "Adaptive tuning to frequency response specifications," Automatica, vol. 29, no. 6, pp. 1557-1563, 1993.

[9] M. N. Ab Malek and M. S. Mohamed Ali, "Evolutionary tuning method for PID controller parameters of a cruise control system using metamodeling," Modelling and Simulation in Engineering, vol. 2009, Article ID 234529, 8 pages, 2009.

[10] L. Wang, X. Fang, S. Duan, and X. Liao, "PID controller based on memristive CMAC network," Abstract and Applied Analysis, vol. 2013, Article ID 510238, 6 pages, 2013.

[11] A. Datta, M. T. Ho, and S. P. Bhattacharyya, Structure and Synthesis of PID Controllers, Springer, New York, NY, USA, 2000.

[12] Q.-G. Wang, Z. Zhang, K. J. Astrom, and L. S. Chek, "Guaranteed dominant pole placement with PID controllers," Journal of Process Control, vol. 19, no. 2, pp. 349-352, 2009.

[13] M. Valasek and N. Olgac, "Efficient pole placement technique for linear time-variant SISO systems," IEE Proceedings: Control Theory and Applications, vol. 142, no. 5, pp. 451-458, 1995.

[14] M. de la Sen, "ON pole-placement controllers for linear timedelay systems with commensurate point delays," Mathematical Problems in Engineering, vol. 2005, no. 1, pp. 123-140, 2005.

[15] J. G. Ziegler and N. B. Nichols, "Optimum settings for automatic controllers," Transactions of the ASME, vol. 64, pp. 759-768, 1942.

[16] K. G. Papadopoulos, K. Mermikli, and N. I. Margaris, “Optimal tuning of PID controllers for integrating processes via the symmetrical optimum criterion," in Proceedings of the 19th Mediterranean Conference on Control and Automation (MED '11), pp. 1289-1294, grc, June 2011.

[17] L. Loron, "Tuning of PID controllers by the non-symmetrical optimum method," Automatica, vol. 33, no. 1, pp. 103-107, 1997.

[18] S. Preitl and R.-E. Precup, "Extension of tuning relations after symmetrical optimum method for PI and PID controllers," Automatica, vol. 35, no. 10, pp. 1731-1736, 1999. 
[19] V. Nicolau, Contributions in advanced automatic control of naval systems [Ph.D. thesis], University of Galati, Galati, Romania, 2004, (Romanian).

[20] T. I. Fossen, Guidance and Control of Ocean Vehicles, John Wiley \& Sons, New York, NY, USA, 1994.

[21] K. Nomoto, "Response analysis of manoeuvrability and its application to ship design," Journal of the Society of Naval Architects of Japan, vol. 11, 1966.

[22] E. Ceanga, C. Nichita, L. Protin, and N. A. Cutululis, Theorie de la Commande des Systemes, Tehnica, Bucharest, Romania, 2001. 


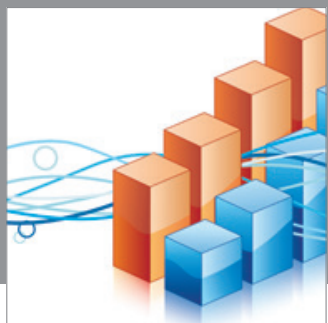

Advances in

Operations Research

mansans

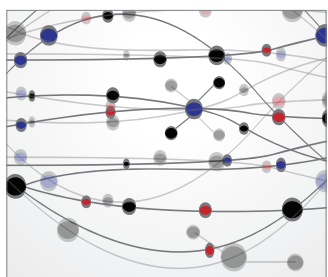

The Scientific World Journal
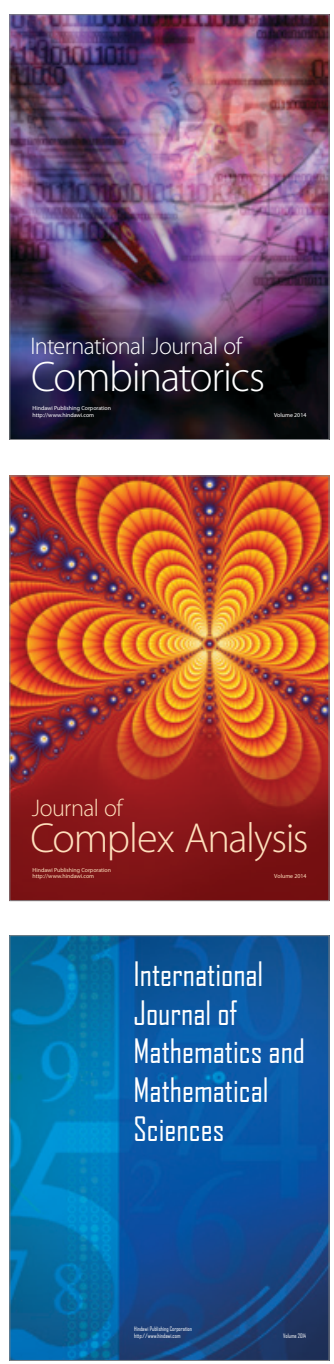
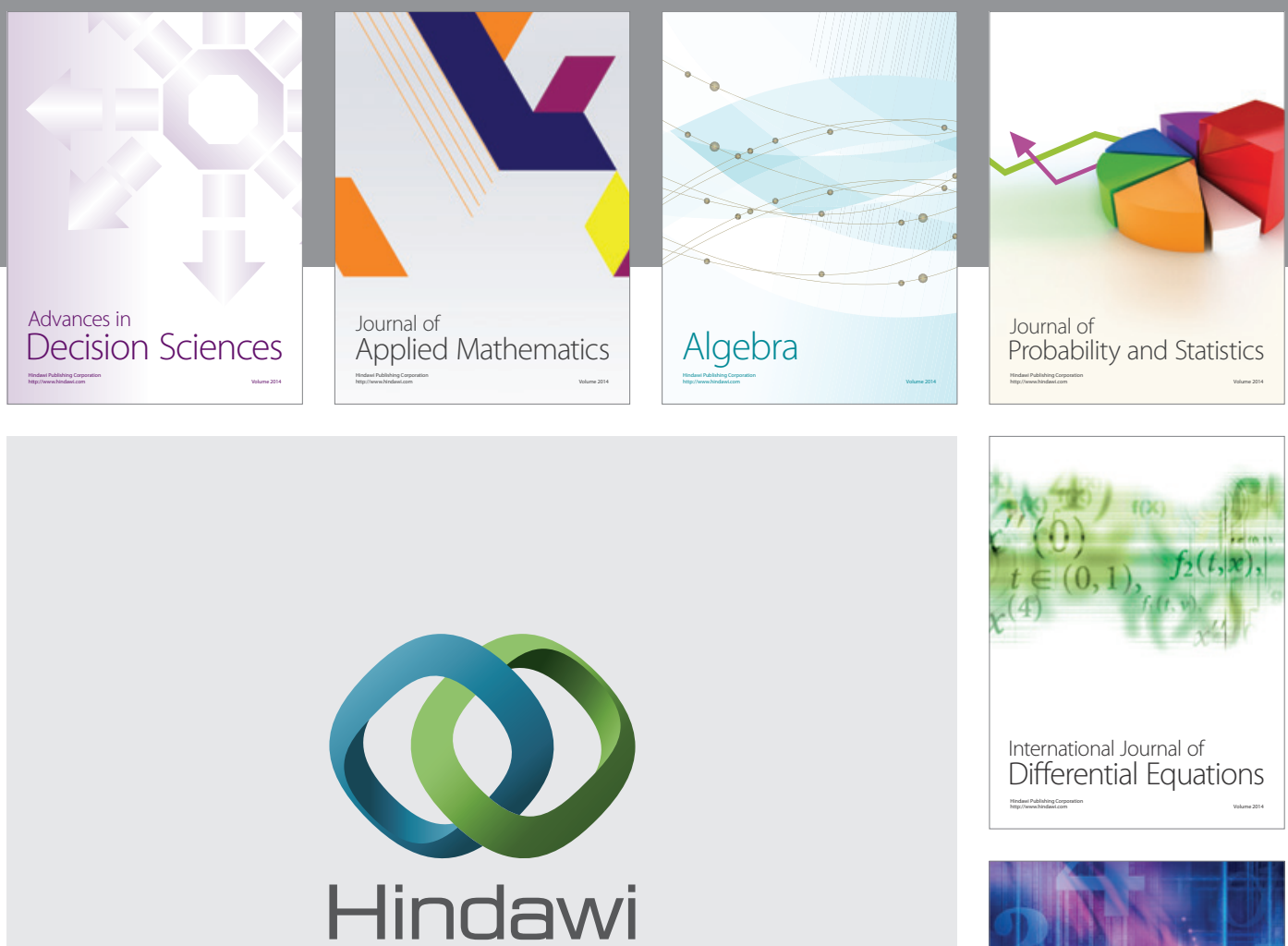

Submit your manuscripts at http://www.hindawi.com
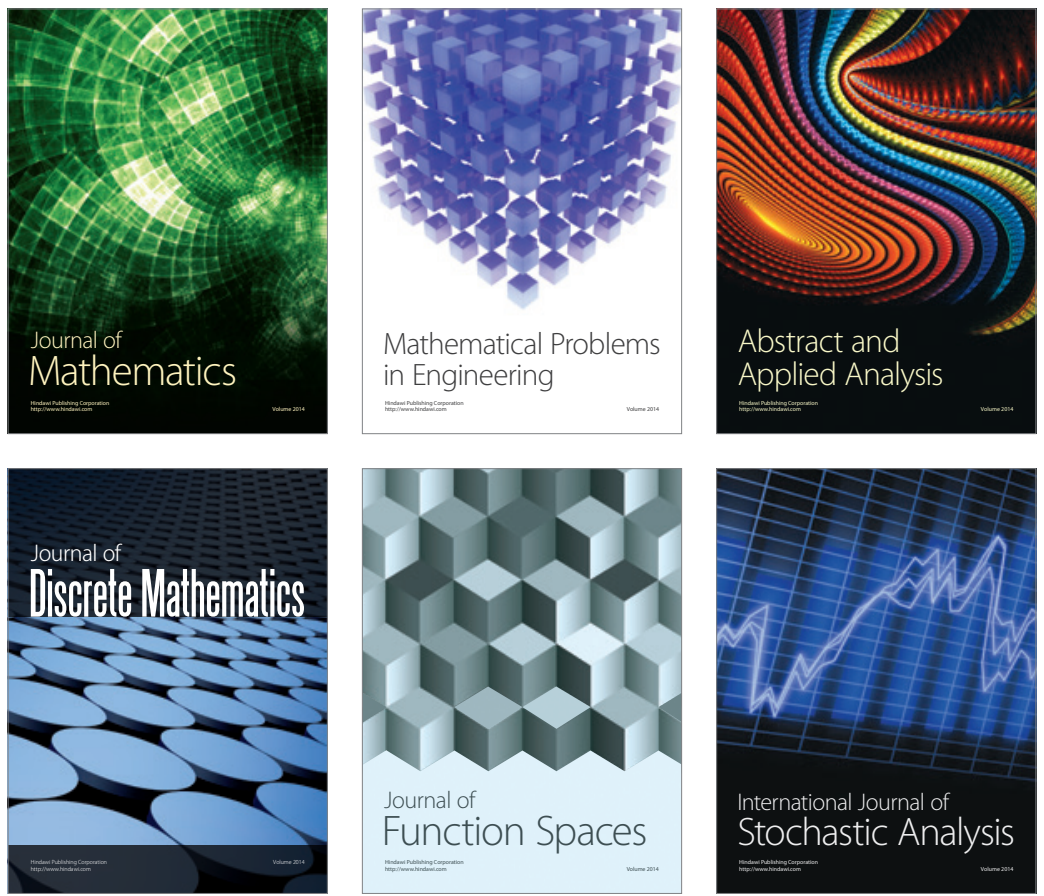

Journal of

Function Spaces

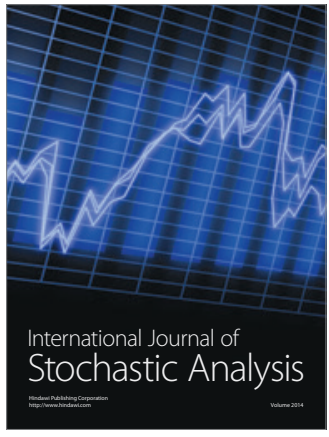

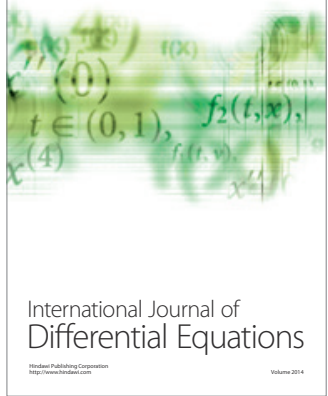
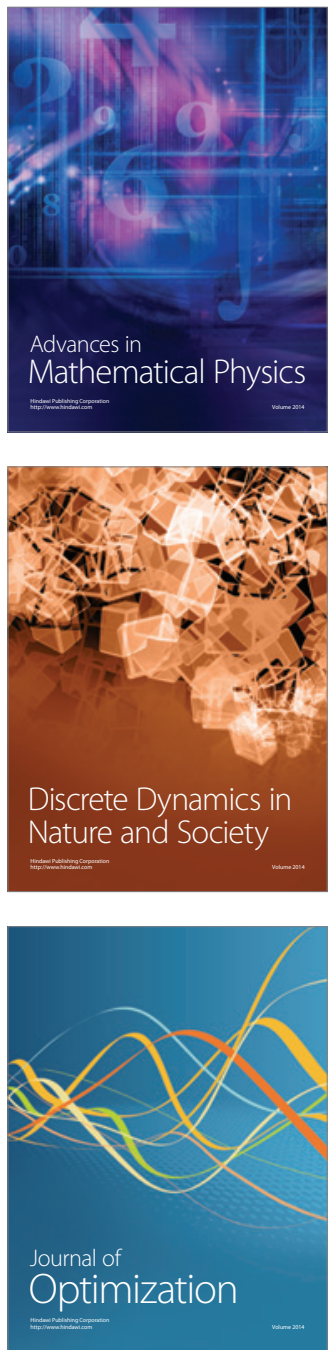